\title{
Et tu Mucor?
}

\author{
Anadi Khatri ${ }^{\mathbb{D} \otimes}$, Gunjan Prasai $^{2}$, Mukesh Pandey $^{3}$, Muna Kharel $^{4}$, Dikshya Bohara ${ }^{5}$, \\ Madhav Panthi ${ }^{6}$, Najara Thapa ${ }^{7}$, Bhaskar Jha ${ }^{8}$, Eli Pradhan ${ }^{9}$ \\ ${ }^{1}$ Birat Eye Hospital, Biratnagar, Nepal \\ ${ }^{2}$ Kathmandu Medical College and Teaching Hospital, Kathmandu, Nepal \\ ${ }^{3}$ Nepal Eye Hospital, Kathmandu, Nepal \\ ${ }^{4}$ Nepal Army Institute of Health Sciences, Kathmandu, Nepal \\ ${ }^{5}$ B.P. Koirala Lions Center for Ophthalmic studies, Kathmandu, Nepal \\ ${ }^{6}$ Birat Medical College and Teaching Hospital, Biratnagar, Nepal \\ ${ }^{7}$ Lumbini Eye Institute and Research Center, Lumbini, Nepal \\ ${ }^{8}$ Bhaskar Eye Care and Glaucoma Center, Biratnagar, Nepal \\ ${ }^{9}$ Tilganga Institute of Ophthalmology, Kathmandu, Nepal
}

\begin{abstract}
Introduction: Recently, the Indian subcontinent has been hard hit by the second wave of COVID-19. Along with it, the region has become a hot spot for mucormycosis infection in patients with COVID-19. The aim of this paper is to provide a scientific overview on the status of mucormycosis in pre-COVID-19 era and on how COVID-19 could have directly or indirectly expose the hidden burden of mucormycosis globally.
\end{abstract}

Materials and methods: This paper is structured as a narrative review and is based on relevant published literature on both COVID-19 and mucormycosis.

Conclusion: Mucormycosis in the pre-COVID-19 era was almost a neglected entity. It was because the risk factors for both - More severe COVID-19 infections and Mucormycosis are almost similar - COVID-19 might have helped to expose the true magnitude of the mucormycosis and flagging it as a major public health concern requiring a revisit by medical science.

Key words: COVID-19, Expose, Mucormycosis, Public health concern.

$\begin{array}{ll}\text { Financial Interest }: \text { Nil } & \text { Received : 21.02.2021 } \\ \text { Conflict of Interest : Nil } & \text { Accepted : 03.05.2021 } \\ \text { Corresponding Author } & \\ \text { Dr. Anadi Khatri } & \\ \text { Birat Eye Hospital, } & \\ \text { Biratnagar, Nepal } & \\ \text { E-mail: anadikc@gmail.com } & \\ \text { Contact: +9779841767205 } & \end{array}$

Financial Interest : N

Contact: +9779841767205
Access this article online

Website: www.nepjol.info/index.php/NEPJOPH DOI: https://doi.org/10.3126/nepjoph.v13i2.39751 Copyright $\odot 2021$ Nepal Ophthalmic Society ISSN: 2072-6805, E-ISSN: 2091-0320

This work is licensed under a Creative Commons Attribution-NonCommercial-NoDerivatives 4.0 International License (CC BY-NC-ND). 


\section{INTRODUCTION}

Increasing reports on the incidence of mucormycosis, commonly known as the "black fungus" in coronavirus disease of 2019 (COVID-19) patients, has been catching the attention of treating physicians and ophthalmologists. Although mostly isolated to India, it has become a major public health concern- especially considering its case fatality rate.

Given the rapidity at which the cases are being reported, we believe it is primarily important to understand how these infections could be interconnected. While it is beyond the scope of this review to comment on associations, correlations or treatment strategies, it tries to summarize the basics and attempts to improve the understanding towards mucormycosis in relation to COVID-19. It also shortly highlights critical, evidence-based reports regarding the possibilities on why these two entities could be both- dependent or mutually exclusive events and also regarding the status of mucormycosis in the region during the pre-COVID era (Soutik B, 2021).

\section{Mucormycosis}

Mucormycosis is an angio-invasive fungal infection with a remarkably high morbidity and mortality. A rise in its incidence has been observed in recent years - which is mainly thought to be due to an increase in the susceptible population living longer, contributed by better understanding of various diseases and advancements in their treatment.

The upsurge has been perceived globally, more alarmingly in the Asian continent (Prakash $\mathrm{H}$ and Chakrabarti A, 2019). While diabetes mellitus (DM) remains the most common cause, posttuberculosis status and chronic renal failure have emerged as new risk groups (Rammaert et al, 2012). The rhino-orbito cerebral mucormycosis (ROCM) predominates in patients with DM, whereas pulmonary mucormycosis is the most common variant in patients with hematological malignancy and solid organ transplant (Jeong et al, 2019). Likewise, cutaneous mucormycosis and isolated renal mucormycosis are mostly seen in immunocompetent patients - with the latter being reported only from China and India.

\section{COVID-19 and Mucormycosis : Exposing the concealed}

The general apprehension worldwide due to the pandemic is being further fueled by the recent reports regarding an increase in the incidence of mucormycosis among patients with COVID-19. There are already several factors which are responsible for the spike in incidence of rhinoorbito-cerebral mucormycosis. These mainly include diabetes, long term use of steroids and immunosuppressants, hematological malignancies, solid organ transplantation, other immunocompromising diseases, malnutrition, inborn errors of hemoglobin and iron metabolism (Sen et al, 2021). 
Not so surprisingly, many of these risk factors are the same as those known to make COVID-19 patients more serious - often requiring intensive care unit admission or mechanical ventilation (Mekonnen et al, 2021). Furthermore, the use of high dose steroids in these patients with risk factors provides an opportunity for favorable growth of ubiquitous filamentous fungi- Mucor (Khatiwada et al, 2012).

\section{Pre-COVID status of mucormycosis}

It is very important to understand that even prior to the COVID-19 outbreak, cases of mucormycosis had been increasingly reported worldwide with a fourfold rise in the last four decades (Prakash H and Chakrabarti A, 2019). In a retrospective study in the United States of America, mucormycosis related hospitalization was found to be 0.12 per 10,00 discharges from January 2005 to June 2014 (Kontoyiannis et al, 2016).

Various countries from Europe too reported cases in accordance to this. In Spain, mucormycosis cases were seen to have increased from a prevalence of 1.2 cases/10,000 admissions between 1988 to 2006 to 3.3 cases/10,000 admissions between 2007 to 2015 (Guinea et al, 2017). France saw its prevalence rate rise from 0.7 cases per million in 1997 to 1.2 per million in 2006 (Bitar et al, 2009) ; Belgium detected a rise from 0.019 cases $/ 10,000$ patient-days in 2000 to 0.148 cases $/ 10,000$ patient-days in 2009(Saegeman et al, 2011); and Switzerland observed a rise in prevalence to 6.3 cases $/ 10,000$ admissions per year from after 2003 while it was 0.57 cases $/ 100,000$ admissions per year before that (Ambrosioni et al, 2010).

Countries in Asia had similar reports as well. Iran saw a rise from $9.7 \%$ in 2008 to $23.7 \%$ in 2014 (Dolatabadi et al, 2018). India had a marked increment- from 24.7 cases per year between 1990 and 2007 almost quadrupling to 89 cases per year between 2013 to 2015 (Prakash et al, 2018). In addition to this, a multicenter study in India has claimed that almost a quarter of the invasive mold infections in India comprises Mucor (Prakash et al, 2019).

Even prior to the COVID-era, the Leading International Fungal Education portal had estimated an international prevalence of 10,000 cases excluding India that astonishingly reached 910,000 when cases in India were included (Chakrabarti, A. and Dhaliwal, M., 2013; Bongomin et al, 2017). Nonetheless, even in these data it must be kept in mind that the true incidence and prevalence of mucormycosis still remain underestimated owing to the fact that the collection of the deep infected tissues are quite problematic and the available diagnostic tests have low sensitivity (Chakrabarti, A. and Dhaliwal, M., 2013).

\section{Risk factors for severe COVID-19 infections and Mucormycosis}

Eight percent of hospitalized COVID-19 patients have been found to develop secondary infections, especially in the middle and later stages. Various meta analytic studies conducted 
independently on COVID-19 and mucormycosis have shown the major risk factors predisposing to both of these conditions. Diabetes mellitus, solid organ transplantation, immunosuppression and malignancies have been distinguished as common to both of these (Rammaert et al, 2012; Geng et al, 2021).

\section{Diabetes Mellitus}

Multiple meta-analysis studies have shown that presence of diabetes mellitus was associated with a greater risk of more critical morbidity, hospital admission and death, more so in those with poor serum glycemic control (de Souza et al, 2021). A COVID patient with hyperglycemia during admission was seen to have more likelihood of needing ICU admission and mechanical ventilation. Furthermore, even new onset hyperglycemia and hyperglycemia during hospitalization were associated with higher mortality (Gao et al, 2021). Adverse outcome among COVID-19 patients was found to be related not only with type 2 diabetes, but also type 1 diabetes (Gregory et al, 2021).

Diabetes mellitus has been seen to be the most common predisposing factor for mucormycosis, ranging from $17 \%$ to $88 \%$ around the world, usually underlying more than half the cases reported in India (Jeong et al, 2019). In the most extensive literature review of mucormycosis ranging from 1885 to 2004 , more than half the cases were observed to be known diabetics and a substantial number had ketoacidosis, almost half of the latter being diagnosed with diabetes mellitus only on admission (Rammaert et al, 2012).

\section{Hematological disorders and malignancies}

People with hematological disorders and malignancies have compromised immunity making them more vulnerable to COVID-19 infection with the severity being attributed to the type of tumor, duration and therapy (Booth et al, 2021). A study in Wuhan showed that cancer patients were more likely to have a grave prognosis (Gao et al, 2021).

Hematological malignancy has been associated with mucormycosis cases also. It lies as an underlying disorder just second to that of diabetes mellitus worldwide and with a poorer prognosis (Jeong et al, 2019). In Europe and the United States of America, these groups have been seen to rank the highest comorbid condition among the reported cases of mucormycosis in multiple studies, ranging from $38 \%$ to $62 \%$ (Rammaert et al, 2012; Lanternier et al, 2012). Boelaert et al. exhibited an accelerated infection with mucormycosis among those with hematological malignancies and hematopoietic stem cell transplantation (Boelaert et al, 1993).

In addition to immunosuppression, such patients require frequent blood transfusions that may cause iron overload and deferoxamine therapy that are known to play an important role in mucormycosis pathogenesis. Rhizopus sp. captures the iron removed by deferoxamine that further potentiates its growth. 


\section{Chronic kidney disease}

Chronic Kidney disease (CKD) has also been found to be associated with higher likelihood of getting COVID-19. A comprehensive prospective cohort study conducted in Wuhan, a high prevalence of chronic kidney disease was observed among hospitalized COVID-19 patients (Cheng et al, 2020). CKD patients have also been scrutinized to have a higher death rate when associated with COVID-19 than those with good renal function. A study in China has shown that patients of End stage renal disease were more likely to die during hospital admission if they contracted COVID-19 in contrast to those who did not get infected with it.(Gao et al, 2021) Dialysis, organ transplantation and End stage renal disease are among the top tier of associated comorbidities causing mortality from COVID-19 (Noordzij et al, 2020).

Mucormycosis has been seen in rise among chronic kidney disease patients in developing countries. Gupta et al. reported nine cases of CKD, two of which had rhino cerebral mucormycosis (Gupta et al , 1989). CKD was observed in $9-32 \%$ of mucormycosis cases in India and 18\% in Turkey (Kursun et al, 2015).

\section{Solidorgantransplant/Immunocompromised state:}

Chronic immunosuppression required pre and post solid organ transplantation keeps such transplant recipients at a higher risk of contracting COVID-19. A huge mortality rate between $13 \%$ to more than $30 \%$ have been seen among COVID-19 patients who had previously undergone solid organ transplantation (Azzi et al, 2021).

Such patients have also been reported to have an increased occurrence of mucormycosis, accounting for $2-15 \%$ of mucormycosis cases. The Transplant-Associated Infection Surveillance Network (TRANSNET) study estimated a $0.07 \%$ 12-month cumulative prevalence of mucormycosis among solid organ recipients (Kontoyiannis et al, 2016).

Other risk factors associated with mucormycosis are HIV infection, intravenous drug use, low birth weight infants, malnutrition, chronic alcoholism, liver diseases, chemotherapy and use of calcineurin inhibitors (Prakash et al, 2018). A recent study from India reported chronic kidney disease $(8.9 \%)$ and postpulmonary mucormycosis $(6.9 \%)$ as emerging risk factors of mucormycosis .

\section{Nosocomial}

Mucormycosis, a rare but serious fungal infection can occur sporadically in the community. In fact, percentage of Rhizopus spores in the hospital air shows $20 \%$ more than outside which is about $5 \%$. There are various documentation supporting hospital related mucormycosis due to use of contaminated wooden depressors, adhesive bandages, umbilical catheters, negative pressure rooms, poor air filtration, water leaks, hospital linens and non-sterile instruments used for patients (Rammaert et al, 2012; Hartnett et al, 2019; 
Petrikkos et al, 2003). One of the hospitals in the United States has documented isolation of Rhizopus species in $42 \%$ of clean linens (Duffy et al, 2014). With the alarming rate of increase in mucormycosis cases and dreadful prognosis related to it, the treating physician should be aware of the nosocomial mucormycosis.

Rampant use of voriconazole in recent years and also use of industrial oxygen cylinders to compensate for the skyrocketing demands during the COVID-19 crisis are also thought to be related with the spike in the Mucor cases although the latter has remained a speculation so far (Skiada et al, 2020).

Mucormycosis can also develop as clusters especially in hospitals where these cases are being treated (Antoniadou et al, 2009). Although non- contagious, the spores of the fungus can remain in the surroundings - especially in the intensive care units or operating rooms. Any susceptible patient being treated in the same environment may contract and have cluster infections. Hence hospitals accepting and treating patients with suspected or confirmed cases of mucormycosis must practice extra precaution to break the chain of transmission.

\section{In Focus: Rhino-Orbito-Cerebral Mucormycosis (ROCM)}

Based on anatomical sites, it can involve many parts of the human body, but Rhino Orbital Cerebral mucormycosis (ROCM) is still the most common form.
Generally, patients who have COVID-19 will have symptoms like fever, fatigue, dyspnea, anosmia and headache which is also similar to the symptoms of mucormycosis. But in mucormycosis, it is not just limited to just these symptoms. Patients will present with symptoms like facial swelling, facial pain, facial numbness, facial nerve palsy, cough, anosmia, distortion/ loss of taste , nasal discharge, nasal obstruction, epistaxis, sinusitis, palatal eschar, toothache, bone discharge and altered mental status ( Prakash et al, 2018). Moreover, ophthalmological symptoms like unilateral eye pain, loss of vision, ophthalmoplegia, proptosis, ptosis, relative afferent pupillary defect, orbital cellulitis and periorbital discoloration and necrosis are observed in cases of mucormycosis.

It is important to use these clues to suspect ROCM and plan for diagnostic imaging or histopathological examinations as the pathology is very rampant and can result in mortality in a matter of a few days if missed.

\section{The Challenge ahead:}

The transmogrification of a pandemic like COVID-19 has grave detriment in a developing country like Nepal, with limited professional manpower, and lack of sufficient infrastructure. The rising incidence of opportunistic infections like mucormycosis in these patients, and also in co-morbid diabetics, makes it more difficult to contain these infections. The diagnosis and management of cases infected with COVID-19 and mucormycosis are way challenging. There 
is a limited sensitivity and specificity of clinical approach to diagnosis. Histopathologic and direct microscopy, added on with culture and molecular assays do help in the diagnosis, but the foremost challenge is the optimal and appropriate suspicion of this infection. Once a timely diagnosis is made, the yet prodigious challenge is the management, which advocates multidisciplinary involvement, which is still in scarce in many of the health institutions across the country. Many patients also require destructive surgeries, which has its own impact on the physical and mental well-being of patients and their families. Another challenge to early diagnosis and aggressive management is the high morbidity and mortality rates, even with the standard of care that is currently being improvised.

With such an unrelenting opportunistic infection in a patient already coping with an inexorable infection like COVID-19, the difficulties that the patient, their families, as well as involved clinicians face, is always calamitous. Still, with efficacious antifungals, and development of standard protocols to treat the disease stagewise, shall regain the confidence of treating physicians and surgeons, and will also provide a ray of hope for the patients and their families.

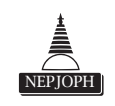

\section{REFERENCES}

Ambrosioni, J., Bouchuiguir-Wafa, K. and Garbino, J., 2010. Emerging invasive zygomycosis in a tertiary care center: epidemiology and associated risk factors. International Journal of Infectious Diseases, 14, pp.e100-e103.

Antoniadou, A., 2009. Outbreaks of zygomycosis in hospitals. Clinical Microbiology and Infection, 15, pp.55-59.

Azzi, Y., Bartash, R., Scalea, J., Loarte-Campos, P. and Akalin, E., 2021. COVID-19 and solid organ transplantation: a review article. Transplantation, 105(1), pp.37-55.

Bitar, D., Van Cauteren, D., Lanternier, F., Dannaoui, E., Che, D., Dromer, F., Desenclos, J.C. and Lortholary, O., 2009. Increasing incidence of zygomycosis (mucormycosis), France, 1997-2006. Emerging infectious diseases, 15(9), p.1395.

Boelaert, J.R., de Locht, M., Van Cutsem, J., Kerrels, V., Cantinieaux, B., Verdonck, A., Van Landuyt, H.W. and Schneider, Y.J., 1993. Mucormycosis during deferoxamine therapy is a siderophore-mediated infection. In vitro and in vivo animal studies. The Journal of clinical investigation, 91(5), pp.1979-1986.

Bongomin, F., Gago, S., Oladele, R.O. and Denning, D.W., 2017. Global and multi-national prevalence of fungal diseases - estimate precision. Journal of fungi, 3(4), p.57.

Booth, A., Reed, A.B., Ponzo, S., Yassaee, A., Aral, M., Plans, D., Labrique, A. and Mohan, D., 2021. Population risk factors for severe disease and mortality in COVID-19: A global systematic review and meta-analysis. PloS one, 16(3), p.e0247461.

Chakrabarti, A. and Dhaliwal, M., 2013. Epidemiology of mucormycosis in India. Current Fungal Infection Reports, 7(4), pp.287-292. 
Cheng, Y., Luo, R., Wang, K., Zhang, M., Wang, Z., Dong, L., Li, J., Yao, Y., Ge, S. and Xu, G., 2020. Kidney impairment is associated with in-hospital death of COVID-19 patients. MedRxiv.

de Souza, F.S.H., Hojo-Souza, N.S., Batista, B.D.D.O., da Silva, C.M. and Guidoni, D.L., 2021. On the analysis of mortality risk factors for hospitalized COVID-19 patients: A data-driven study using the major Brazilian database. PloS one, 16(3), p.e0248580.

Dolatabadi, S., Ahmadi, B., Rezaei-Matehkolaei, A., Zarrinfar, H., Skiada, A., Mirhendi, H., Nashibi, R., Niknejad, F., Nazeri, M., Rafiei, A. and Gharaghani, M., 2018. Mucormycosis in Iran: A six-year retrospective experience. Journal de mycologie medicale, 28(2), pp.269-273.

Duffy, J., Harris, J., Gade, L., Sehulster, L., Newhouse, E., O’Connell, H., Noble-Wang, J., Rao, C., Balajee, S.A. and Chiller, T., 2014. Mucormycosis outbreak associated with hospital linens. The Pediatric infectious disease journal, 33(5), pp.472-476.

Gao, Y.D., Ding, M., Dong, X., Zhang, J.J., Kursat Azkur, A., Azkur, D., Gan, H., Sun, Y.L., Fu, W., Li, W. and Liang, H.L., 2021. Risk factors for severe and critically ill COVID-19 patients: a review. Allergy, 76(2), pp.428-455.

Geng, M.J., Wang, L.P., Ren, X., Yu, J.X., Chang, Z.R., Zheng, C.J., An, Z.J., Li, Y., Yang, X.K., Zhao, H.T. and Li, Z.J., 2021. Risk factors for developing severe COVID-19 in China: an analysis of disease surveillance data. Infectious diseases of poverty, 10(1), pp.1-10.

Gregory, J.M., Slaughter, J.C., Duffus, S.H., Smith, T.J., LeStourgeon, L.M., Jaser, S.S., McCoy, A.B., Luther, J.M., Giovannetti, E.R., Boeder, S. and Pettus, J.H., 2021. COVID-19 severity is tripled in the diabetes community: a prospective analysis of the pandemic's impact in type 1 and type 2 diabetes. Diabetes Care, 44(2), pp.526-532.

Guinea J, Escribano P, Vena A, et al. Increasing incidence of mucormycosis in a large Spanish hospital from 2007 to 2015: Epidemiology and microbiological characterization of the isolates [published correction appears in PLoS One. 2020 Feb 12;15(2):e0229347]. PLoS One. 2017;12(6):e0179136. Published 2017 Jun 7. doi:10.1371/journal. pone. 0179136

Gupta, K.L., Radotra, B.D., Sakhuja, V., Banerjee, A.K. and Chugh, K.S., 1989. Mucormycosis in patients with renal failure. Renal failure, 11(4), pp.195-199.

Hartnett, K.P., Jackson, B.R., Perkins, K.M., Glowicz, J., Kerins, J.L., Black, S.R., Lockhart, S.R., Christensen, B.E. and Beer, K.D., 2019. A guide to investigating suspected outbreaks of mucormycosis in healthcare. Journal of Fungi, 5(3), p.69.

Jeong, W., Keighley, C., Wolfe, R., Lee, W.L., Slavin, M.A., Kong, D.C.M. and Chen, S.A., 2019. The epidemiology and clinical manifestations of mucormycosis: a systematic review and meta-analysis of case reports. Clinical Microbiology and Infection, 25(1), pp.26-34.

Khatiwada, P., Giri, A. and Khatiwoda, P., 2012. Mucormycosis in diabetes mellitus. Journal of Advances in Internal Medicine, 1(2), pp.73-75.

Kontoyiannis, D.P., Yang, H., Song, J., Kelkar, S.S., Yang, X., Azie, N., Harrington, R., Fan, A., Lee, E. and Spalding, J.R., 2016. Prevalence, clinical and economic burden of mucormycosis-related hospitalizations in the United States: a retrospective study. BMC infectious diseases, 16(1), pp.1-6.

Kursun, E., Turunc, T., Demiroglu, Y.Z., Alışkan, H.E. and Arslan, A.H., 2015. Evaluation of 28 cases of mucormycosis. Mycoses, 58(2), pp.82-87. 
Lanternier, F., Dannaoui, E., Morizot, G., Elie, C., Garcia-Hermoso, D., Huerre, M., Bitar, D., Dromer, F., Lortholary, O. and French Mycosis Study Group, 2012. A global analysis of mucormycosis in France: the RetroZygo Study (2005-2007). Clinical Infectious Diseases, 54(suppl_1), pp.S35-S43.

Mekonnen, Z.K., Ashraf, D.C., Jankowski, T., Grob, S.R., Vagefi, M.R., Kersten, R.C., Simko, J.P. and Winn, B.J., 2021. Acute invasive rhino-orbital mucormycosis in a patient with COVID-19-associated acute respiratory distress syndrome. Ophthalmic plastic and reconstructive surgery, 37(2), p.e40.

Noordzij M, Duivenvoorden R, Pena MJ, de Vries H, Kieneker LM; collaborative ERACODA authors. ERACODA: the European database collecting clinical information of patients on kidney replacement therapy with COVID-19. Nephrol Dial Transplant. 2020;35(12):2023-2025. doi:10.1093/ndt/gfaa179

Petrikkos, G., Skiada, A., Sambatakou, H., Toskas, A., Vaiopoulos, G., Giannopoulou, M. and Katsilambros, N., 2003. Mucormycosis: ten-year experience at a tertiary-care center in Greece. European Journal of Clinical Microbiology and Infectious Diseases, 22(12), pp.753-756.

Prakash, H. and Chakrabarti, A., 2019. Global epidemiology of mucormycosis. Journal of Fungi, 5(1), p.26.

Prakash, H., Ghosh, A.K., Rudramurthy, S.M., Singh, P., Xess, I., Savio, J., Pamidimukkala, U., Jillwin, J., Varma, S., Das, A. and Panda, N.K., 2019. A prospective multicenter study on mucormycosis in India: Epidemiology, diagnosis, and treatment. Medical mycology, 57(4), pp.395-402.

Rammaert, B., Lanternier, F., Poirée, S., Kania, R. and Lortholary, O., 2012. Diabetes and mucormycosis: a complex interplay. Diabetes \& metabolism, 38(3), pp.193-204.

Rammaert, B., Lanternier, F., Zahar, J.R., Dannaoui, E., Bougnoux, M.E., Lecuit, M. and Lortholary, O., 2012. Healthcare-associated mucormycosis. Clinical Infectious Diseases, 54(suppl_1), pp.S44-S54.

Saegeman, V., Maertens, J., Meersseman, W., Spriet, I., Verbeken, E. and Lagrou, K., 2010. Increasing incidence of mucormycosis in University Hospital, Belgium. Emerging infectious diseases, 16(9), p.1456.

Sen, M., Lahane, S., Lahane, T.P., Parekh, R. and Honavar, S.G., 2021. Mucor in a viral land: a tale of two pathogens. Indian journal of ophthalmology, 69(2), p.244.

Skiada, A., Pavleas, I. and Drogari-Apiranthitou, M., 2020. Epidemiology and diagnosis of mucormycosis: an update. Journal of Fungi, 6(4), p.265.

Soutik, B., Mucormycosis: The "black fungus" maiming covid patients in India. BBC News. 9 May 2021. 